\title{
Screening for fetal malformations using ultrasound and measurements of $\alpha$-fetoprotein in maternal serum
}

\author{
P H PERSSON，S KULLANDER，G GENNSER， L GRENNERT，C B LAURELL
}

\begin{abstract}
Fetal ultrasound combined with semiquantitative measurements of $\alpha$-fetoprotein in maternal serum was used for early detection of neural tube defects and omphalocele in 10147 pregnancies. The accurate assessment of gestational age, obtained by ultrasound, facilitated evaluation of $\alpha$-fetoprotein concentrations in selecting cases for amniocentesis. The advantage of screening with two independent methods is suggested by the finding that eight out of 10 cases with malformations (spina bifida, encephalocele, anencephalus, omphalocele) were detected when both methods were used. Screening by routine ultrasound alone detected only four malformations and by measurement of $\alpha$-fetoprotein alone only seven.

The results suggest that, in a low risk population, ultrasound should be combined with the measurement of $\alpha$-fetoprotein in screening for neural tube defects. Measurement of $\alpha$-fetoprotein is indispensable in detection of the small neural tube defects, where the fetus would survive with severe sequelae. The semiquantitative analysis of $\alpha$-fetoprotein that may be used in combination with ultrasound examination is of negligible cost.
\end{abstract}

\section{Introduction}

Regular screening programmes with measurements of the $\alpha$-fetoprotein concentration in maternal serum have been

\footnotetext{
University of Lund, Malmö Allmänna Sjukhus, S-214 01 Malmö, Sweden

P H PERSSON, MD, associate professor, department of obstetrics and gynaecology

$S$ KULLANDER, MD, professor, chairman, department of obstetrics and gynaecology

G GENNSER, MD, associate professor, department of obstetrics and gynaecology

L GRENNERT, MD, senior registrar, department of obstetrics and gynaecology

C B LAURELL, MD, professor, department of clinical chemistry
}

initiated in the United Kingdom-and several other countriesfor early antenatal detection of neural tube defects. The relatively high incidence of neural tube defects (about 4.5/1000 births $)^{1}$ in the United Kingdom justified a screening programme. In Sweden the incidence of neural tube defects is lower $(0 \cdot 7 / 1000$ births). ${ }^{2}$ It is unlikely that the incidence of raised maternal serum concentrations of $\alpha$-fetoprotein due to causes other than fetal neural tube defects would show the same regional variation as the incidence of neural tube defects. The proportion of fetuses with neural tube defects among pregnancies in which the mother had raised blood concentrations of $\alpha$-fetoprotein is therefore, as expected, much lower in Sweden than in the United Kingdom. This increases the risk of false interpretation, which could easily reverse the value of such screening in a low risk population. The screening must therefore be highly accurate to obtain a reasonable cost benefit balance. For the past eight years all pregnant women in our community have undergone ultrasound examination in early gestation, primarily to assess gestational age and to detect multiple pregnancies. Correct assessment of gestational age ( $\mathrm{SD} \pm 5$ days) ${ }^{3}$ offered by general ultrasound fetometry was expected to improve the accuracy of the screening for neural tube defects.

We evaluated our findings over 44 months of screening for neural tube defects with ultrasound and measurement of maternal serum $\alpha$-fetoprotein concentrations in low risk pregnant women.

\section{Patients and methods}

All pregnant women in the city of Malmö (240 000 inhabitants) are booked in for screening with ultrasound and measurement of serum $\alpha$-fetoprotein concentration at their first visit in the antenatal care programme. The women are given oral and written information about the screening for neural tube defects and the consequences of raised $\alpha$-fetoprotein concentrations. If they do not want their pregnancy terminated in the event of a fetal malformation being detected they are recommended to abstain from testing of $\alpha$-fetoprotein.

The first routine ultrasound examination is scheduled to take place in the 17th gestational week, judged from last menstrual period and the early clinical assessment of uterine size. Provided the fetal biparietal diameter is between 35 and $49 \mathrm{~mm}$, the blood sample for measurement of $\alpha$-fetoprotein concentration is taken in conjunction with the ultrasound examination. This chosen interval for biparietal diameter corresponds to a gestational age between 15.5 and 20 weeks. 
If the biparietal diameter is below $35 \mathrm{~mm}$ the sampling for $\alpha$-fetoprotein concentration is postponed until the biparietal diameter would be expected to be $37 \mathrm{~mm}$ (corresponding to, on average, 16.8 weeks). In cases where the biparietal diameter exceeds $49 \mathrm{~mm}$ the analysis of $\alpha$-fetoprotein is not performed. Women with a multiple pregnancy or a dead fetus detected at the ultrasound scanning are excluded from the measurement of $\alpha$-fetoprotein.

During the study period determination of $\alpha$-fetoprotein concentrations in maternal serum was performed weekly using a countercurrent immunoelectrophoretic technique. ${ }^{4}$ A $40 \mu 1$ sample of serum was placed in a circular well of $7 \mathrm{~mm}$ diameter at a distance of $10 \mathrm{~mm}$ from a $10 \times 1 \mathrm{~mm}$ antiserum slit filled with $10 \mu \mathrm{l}$ rabbit antihuman $x$-fetoprotein. The electrophoresis was run for $5 \mathrm{~h}$ with $2 \mathrm{~V} / \mathrm{cm}$ without cooling. The $1 \mathrm{~mm}$ thick agarose gel rested on a $1.5 \mathrm{ml}$ thick glass $(110 \times 210 \mathrm{~mm})$ plate. Three standard sera with 200,100 , and $50 \mu \mathrm{g} \alpha$-fetoprotein/l were run in each row of samples. Two rows of samples were run on each plate (24 samples + standards). The intensity of the precipitate formed was compared by naked eye with the standards, and the results were reported as less than $100 \mu \mathrm{g}$ $\alpha$-fetoprotein/l, about 100 , or above 100 . The antiserum was diluted so that precipitates obtained with serum containing $50 \mu \mathrm{g} \alpha$-fetoprotein/l were just observable. On average, time taken for analysis of $\alpha$-fetoprotein was $3 \mathrm{~min} / \mathrm{sample}$. Based on a preliminary study on 1000 subjects and on published reports, a cut off value of about 100 $\mu \mathrm{g} \alpha$-fetoprotein/l was chosen to differentiate between raised and normal concentrations. Each woman with serum $\alpha$-fetoprotein concentration above $100 \mu \mathrm{g} / \mathrm{l}$ was scheduled for amniocentesis. Concentration of $\alpha$-fetoprotein in amniotic fluid was analysed with electroimmunoassay ${ }^{5}$ and reference values published by NörgaardPedersen were used. ${ }^{6}$ Amniotic fluid concentrations of $\alpha$-fetoprotein greater than three times the median value for the gestational age were considered indicative of fetal malformation. Only if a malformation could be confirmed by a most careful repeated ultrasound examination was termination recommended.

The maximum time taken from the initial blood sampling to the result of the analysis was one week. The result of the measurement of $\alpha$-fetoprotein in amniotic fluid was available within one day.

From 10 April 1978 to 31 December 1981 a total of 10147 pregnant women underwent routine ultrasound examination. Of these, 8610 had serum concentrations of $\alpha$-fetoprotein analysed. A total of 1537 women were excluded from analysis of $\alpha$-fetoprotein: 297 of these abstained; 86 were excluded because ultrasound showed a twin pregnancy, and 70 because it showed the fetus to be dead. The remaining 1084 women had their first examination after 19 weeks' gestation. In 1041 cases where fetal biparietal diameter was less than $35 \mathrm{~mm}$ a blood sample was taken at a later visit. A follow-up on the outcome of pregnancy with regard to congenital malformations and late abortions was carried out on all women examined by ultrasound. Seventy women aborted spontaneously after normal findings on ultrasound and analysis of serum $\alpha$-fetoprotein concentration. Two spontaneous abortions occurred within 3 weeks of amniocentesis.

\section{Results}

Altogether 10 malformations that have been reported to raise serum concentrations of $\alpha$-fetoprotein occurred: five open spina bifida, three encephalocele, one anencephalus, and one omphalocele, of which eight were detected in the combined screening programme. Tables I and II show the efficiency of the separate methods.

One hundred and twenty women had serum $\alpha$-fetoprotein concentrations above $100 \mu \mathrm{g} / 1$. The prevalence of malformation was $6.7 \%$ in this group. Two false-negatives occurred in measurement of serum $\alpha$-fetoprotein concentrations: in one case of spina bifida the concen-

TABLE I-Efficiency of tests used in screening for neural tube defects. Figures are numbers of women tested

\begin{tabular}{crrrr}
\hline & $\begin{array}{c}\text { Serum } \\
\alpha \text {-fetoprotein }\end{array}$ & Ultrasound & $\begin{array}{c}\text { Amniotic } \\
\text { fluid } \\
\alpha \text {-fetoprotein* }\end{array}$ & $\begin{array}{c}\text { Combined } \\
\text { tests }\end{array}$ \\
\hline $\begin{array}{c}\text { True positive } \\
\text { False positive }\end{array}$ & 8 & 4 & 7 & 8 \\
True negative & 112 & 0 & 2 & 0 \\
False negative & 8488 & 10137 & 106 & 10137 \\
\hline Total & 2 & 6 & 2 & 2 \\
\hline
\end{tabular}

* Measured only in women with raised serum concentrations $(\cdots 100 \mathrm{ug} / \mathrm{l})$.
TABLE II-Sensitivity, specificity, and predictive values of the four different methods used to detect fetal malformations (neural tube defects and omphalocele)

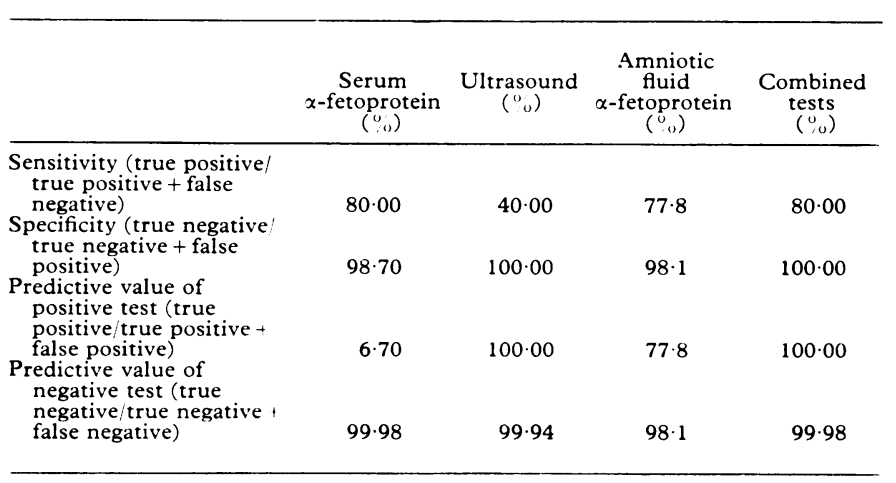

tration was about $100 \mu \mathrm{g} / \mathrm{l}$ (borderline value) and no further action was taken as routine ultrasound failed to detect the lesion. In the other case an encephalocele was detected by ultrasound but the amniocentesis showed $\alpha$-fetoprotein concentrations below the 5 th centile.

Amniocentesis was performed in 117 of the 120 cases of raised serum $\alpha$-fetoprotein concentrations. Amniocentesis was not performed in three cases: in two pregnancies the fetus had succumbed after the ultrasound examination and the sampling for measurement of serum $\alpha$-fetoprotein, and one woman wanted to terminate the pregnancy before amniocentesis as the routine ultrasound examination showed a grossly malformed fetus with encephalocele. The figure shows the concentrations of $\alpha$-fetoprotein in amniotic fluid of the 117 women. Nine women had a value greater than three times the median: in three of these the primary routine ultrasound screening had revealed a fetal malformation and in four women the repeated focused ultrasound examination showed a fetal malformation. When these seven pregnancies were terminated six fetuses had neural tube defects and one

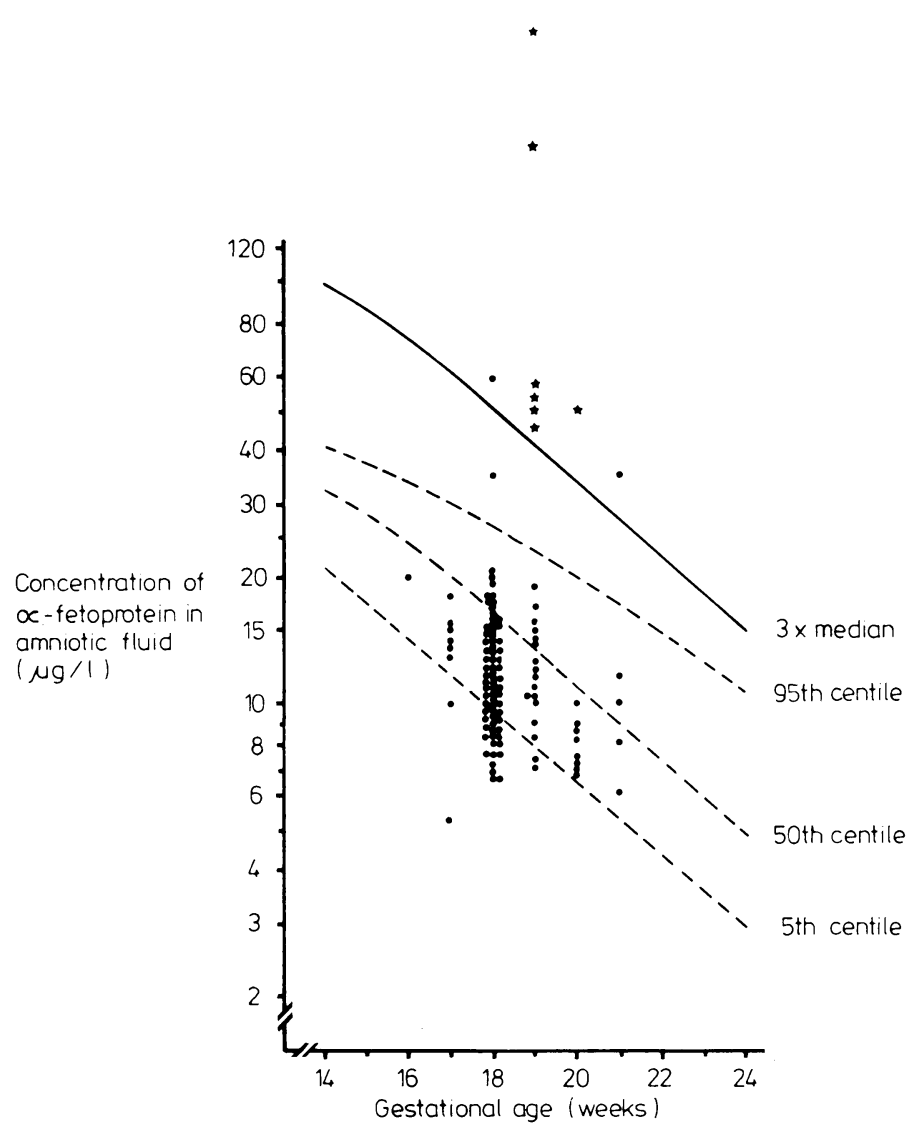

Concentration of $\alpha$-fetoprotein in amniotic fluid of 117 women with raised serum concentrations. Values are plotted in reference distribution of Nörgaard-Pedersen (ref 6). Stars represent concentrations for malformed fetuses and dots for non-affected fetuses. The cut off value was set to $3 \times$ median. 
omphalocele. The remaining two pregnant women, who had $\alpha$-fetoprotein concentrations in amniotic fluid 3-3.5 times that of the median were not recommended to interrupt their pregnancies, as ultrasound did not show any signs of fetal malformation; both women delivered perfectly normal infants at term. One woman had raised serum concentration of $\alpha$-fetoprotein but amniotic fluid concentrations within normal ranges and normal appearance on repeat focused ultrasound examination. This pregnancy was not interrupted: a baby was born with a small meningocele, and survived without severe handicaps.

Eight out of nine fetal defects could be visualised by careful ultrasound scanning. Six were, however, not detected until raised maternal serum concentrations of $\alpha$-fetoprotein gave rise to the suspicion of a malformation.

One hundred and seven women with raised serum concentrations of $\alpha$-fetoprotein and no indication of fetal malformation continued their pregnancy. Table III shows the increased prevalence of complications in the second half of gestation in this group. Moreover, $24 \%$ of the women (mean age 26.8 years) had experienced a previous spontaneous abortion compared to $12 \%$ in the women with normal concentrations of $\alpha$-fetoprotein (mean age 24.6 years) and $17 \%$ had reported vaginal haemorrhage in the first trimester of the present pregnancy compared with $7 \%$ in the control group. In 19 of the 117 women who had amniocentesis performed, the amniotic fluid showed a brownish miscolour, indicating a previous intra-amniotic haemorrhage.

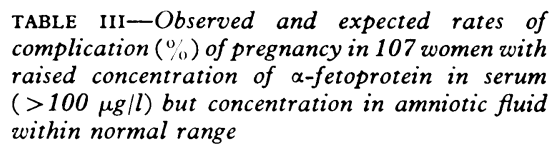

\begin{tabular}{lcc}
\hline & & \\
\hline Preterm delivery & Observed & Expected \\
Birth weight \$5th centile & 12 & $6 \cdot 0$ \\
Ablatio placentae & 14 & $5 \cdot 0$ \\
Perinatal deaths & 4 & $0 \cdot 3$ \\
Late abortions & 5 & $1 \cdot 0$ \\
& 5 & $0 \cdot 7$ \\
\hline
\end{tabular}

\section{Discussion}

Both ultrasound and measurement of serum concentrations of $\alpha$-fetoprotein fulfilled the criteria of good screening methods. The predictive value of a negative test is very high. The number whose risk is detected by measurements of serum $\alpha$-fetoprotein (all positive results) was small and could be handled clinically with the application of other diagnostic tests (ultrasound, estimates of concentration of $\alpha$-fetoprotein amniotic fluid, or both). It has recently been suggested that ultrasound alone could replace measurement of $\alpha$-fetoprotein concentrations in serum in screening for neural tube defects. In this study the primary ultrasound examination alone had a low sensitivity; the malformations detected by this method were all of the severe type that would not survive extrauterine life. Only four of the fetuses would have survived delivery with severe sequelae: screening of $\alpha$-fetoprotein concentration is necessary to detect these small defects. If a qualified repeat focused ultrasound examination had not been performed, however, two falsepositive $\alpha$-fetoprotein values in amniotic fluid would have led to termination of pregnancy. Furthermore, amniocentesis caused spontaneous abortions in two cases. For these reasons it is likely that the negative effects of amniocentesis and $\alpha$-fetoprotein screening would have outweighed the positive effects.

Ultrasound fetometry provides reliable assessment of gestational age, which is the prerequisite for using a semiquantitative assay to measure maternal serum concentrations of $\alpha$-fetoprotein. This method, discriminating values below or above a given cut off point, is useful only when the blood samples are obtained within a rather short period of gestation. The total cost for the ultrasound examination is at present $£ 7$ per examination. The addition of measurement of concentrations of $\alpha$-fetoprotein using the immunoelectrophoretic method adds less than $50 p$ to this sum. Screening with commercially available radioimmunoassay kits alone costs between $£ 3$ and $£ 5$, depending on the work load and the facilities available in the laboratory. Apart from the economic gain, the immunoelectrophoretic method has the advantage of fewer false-positive results than some other reported methods ${ }^{2}$ and also of making repeat tests unnecessary.

The chance observation that raised serum concentrations of $\alpha$-fetoprotein signal fetal disorders other than neural tube defects was confirmed in this study. ${ }^{2}{ }^{7}$ Women with raised serum concentrations of $\alpha$-fetoprotein and normal amniotic fluid concentrations run an increased risk of complication during pregnancy. Increased antenatal surveillance should be directed to this group of pregnant women.

It is our opinion that, in a low risk population, measurement of $\alpha$-fetoprotein concentrations to detect fetuses with neural tube defects should be avoided without qualified ultrasound facilities. Screening by measurement of $\alpha$-fetoprotein concentration alone in a low risk population has a lower priority than screening by general ultrasound. The evaluation of serum $\alpha$-fetoprotein concentrations is made safer when correct gestational age is obtained with fetometry, and, by using the immunoelectrophoretic method, measurement of $\alpha$-fetoprotein concentrations may be used with ultrasound in screening with low additional cost. In cases of raised serum $\alpha$-fetoprotein concentration a focused ultrasound examination may replace the amniocentesis in most cases, but ultrasound examination alone is insufficiently sensitive to detect small neural tube defects.

Few women in our study had raised serum concentrations of $\alpha$-fetoprotein, and false results are avoided by the combination of reliable estimation of gestational age and the use of the semiquantitative analysis of $\alpha$-fetoprotein concentrations. Furthermore, the period from first notifying the mother of a raised concentration to the final result of the subsequent examination is very short-not longer than two days-which reduces any anxiety caused to the mother by the screening procedure. Lack of false results in a screening is the best way to reduce anxiety over fetal malformations among pregnant women.

\section{References}

1 UK Collaborative Study on Alpha-fetoprotein in Relation to Neural-tube Defects. Maternal serum-alpha-fetoprotein measurement in antenatal screening for anencephaly and spina bifida in early pregnancy. Lance $1977 ; \mathrm{i}: 1323-32$

${ }^{2}$ Kjessler B, Johansson SGD. Monitoring of the development of early pregnancy by determination of alpha-fetoprotein in maternal serum and amniotic fluid samples. Acta Obstet Gynecol Scand (Suppl) 1977;69:5-14.

3 Persson PH, Grennert L, Gennser G, Gullberg B. Normal range curve for the intrauterine growth of the biparietal diameter. Acta Obstet Gynecol Scand (Suppl) 1978;78:15-20.

4 Kohn J. Method for the detection and identification of alpha fetoprotein in serum. F Clin Pathol 1970;23:733-5.

${ }^{5}$ Laurell CB. Quantitative estimation of proteins by electrophoresis in agarose gel containing antibodies. Anal Biochem 1966;15:45-52.

${ }^{6}$ Nörgaard-Pedersen B. Human alpha-fetoprotein. Scand $\mathfrak{f}$ Immunol (Suppl) 1976;4:1-45.

7 Wald N, Cuckle H, Stirrat GM, Bennet MJ, Turnbull AC. Maternal serum-alpha-fetoprotein and low birth-weight. Lancet 1977;ii:268-70.

(Accepted 18 November 1982)

ONE HUNDRED YEARS AGO The latest proposal for the promotion of temperance is one which aims at the establishment of a Violet Ribbon Army-an organisation specially designed to put a stop to the practice of taking "nips" by persons engaged in commercial pursuits. The members are to pledge themselves never to take any kind of intoxicating liquors in public-houses or dining-rooms To this, however, should be added a pledge to abstain from indulging in or offering to others "nips" during business hours in city offices In a good many offices, we are told, a decanter of wine is prominent on the mantelpiece or side table for callers to help themselves. This pernicious practice should be treated by "nipping it in the bud." (British Medical fournal 1883;i:468.) 